\title{
A Note on the Generalized Nonlinear Vector Variational-Like Inequality Problem
}

\author{
Ankit Gupta ${ }^{(D)},{ }^{1}$ Satish Kumar, ${ }^{2}$ Ratna Dev Sarma, ${ }^{3}$ Pankaj Kumar Garg, ${ }^{3}$ \\ and Reny George iD ${ }^{4}$ \\ ${ }^{1}$ Department of Mathematics, Bharati College (University of Delhi), Delhi 110058, India \\ ${ }^{2}$ Department of Mathematics, University of Delhi, Delhi 110007, India \\ ${ }^{3}$ Department of Mathematics, Rajdhani College (University of Delhi), Delhi 110015, India \\ ${ }^{4}$ Department of Mathematics, College of Science and Humanities in Al-Kharj, Prince Sattam Bin Abdulaziz University, \\ Al-Kharj 11942, Saudi Arabia
}

Correspondence should be addressed to Reny George; renygeorge02@yahoo.com

Received 30 April 2021; Revised 1 July 2021; Accepted 7 July 2021; Published 28 July 2021

Academic Editor: Yuanfang Ru

Copyright (C) 2021 Ankit Gupta et al. This is an open access article distributed under the Creative Commons Attribution License, which permits unrestricted use, distribution, and reproduction in any medium, provided the original work is properly cited.

In this paper, we discuss two variants of the generalized nonlinear vector variational-like inequality problem. We provide their solutions by adopting topological approach. Topological properties such as compactness, closedness, and net theory are used in the proof. The admissibility of the function space topology and KKM-Theorem have played important role in proving the results.

\section{Introduction}

Variational inequalities have appeared as a working and important tool to investigate various fields of mathematics as well as of sciences including elasticity, vector equilibrium problems, and optimization problems [1-4]. In mid-sixties, Browder [5] formulated and proved the basic existence results for the solutions to a class of nonlinear variational inequality problems. He used a reflexive Banach space $X$ and a monotone nonlinear map $T$ from the space $X$ to its dual space $X^{*}$, to set up the nonlinear variational inequality problem. Browder used the property of hemicontinuity and monotonicity of mapping $T$ along with the lower semicontinuity of $f$, for providing the existence of the solution of nonlinear variational inequality problem. After that, this problem has been generalized and extended in various directions under different set-ups using different techniques. Liu et al. [6], Zhao et al. [7], and Ahmad and Irfan [8] are a few, who extended Browder's results to more generalized nonlinear variational inequalities. In 2009, Farajzadeh et al. [9] considered new kinds of generalized variational-like inequality problems under the frame work of topological vector spaces.
In the subsequent period, generalized quasi-variational inequalities were studied by Hung and others [10-12]. In 2017, Irfan et al. introduced a new generalized variationallike inclusion problem involving relaxed monotone operators [13]. A class of $\eta$-generalized operator variational-like inequalities were introduced by Kim et al. in 2018 [14]. In the same year, Tavakoli et al. studied the C-pseudomonotone property for the set-valued mappings in order to solve a generalized variational inequality problems [15]. On the other hand, vector equilibrium problems for the set-valued mappings were studied by Farajzadeh et al. and Chen et al. during this period $[16,17]$. This wide range of literature is a clear indication of the importance that variational inequality problems have gained in the recent years. In this paper, we further add to this literature by providing solutions to a generalized nonlinear vector variational-like inequality problem, using topological methods.

Variational-like inequalities have number of applications which make it an interesting discipline for research. Vector variational inequality on flow equilibrium problem on a network has been discussed in [18]. Application of variationallike inequality in fuzzy optimization problem is discussed in [19]. More such studies are available in the literature [20-22]. 
Motivated by these studies, here, we investigate a generalized nonlinear variational like inequality problem, which was proposed by Farajzadeh et al. [9] as follows:

Generalized nonlinear variational-like inequality problem: let $\left\langle X, X^{*}\right\rangle$ be a dual system of Hausdorff topological vector spaces and $K$ be a nonempty convex subset of $X$. Given the mappings $f, g, p: X^{*} \longrightarrow X^{*}$ and $\eta: K \times K \longrightarrow$ $X$, set-valued map $M, S, T: K \longrightarrow X^{*}$, and a map $h: K \times K$ $\longrightarrow \mathbb{R}$, consider the following generalized nonlinear variational-like inequality problem (GNVLIP)

$$
\left\{\begin{array}{l}
\text { Find } x \in K \text { such that for each } y \in K, \\
\exists u \in M(x), v \in S(x), w \in T(x) \text { satisfying } \\
\langle p(u)-(f(v)-g(w)), \eta(y, x)\rangle \geq h(x, y)
\end{array}\right.
$$

In this paper, we consider two variants of nonlinear vector variational-like inequality problems in a more general setup as follows:

Let $X$ and $Y$ be two topological vector spaces, and let $K$ be a nonempty, closed, and convex subset of $X$ and $C L(X, Y)$ be the space of all continuous linear mappings from the space $X$ to the space $Y$. Clearly, $C L(X, Y)$ is nonempty as the zero mapping, that is, $k: X \longrightarrow Y$ defined as $k(x)=\mathbf{0}$ for all $x \in X$ is always linear and continuous, hence belongs to $C L(X, Y)$.

Further, let $M, S, T: K \longrightarrow C L(X, Y)$ be set-valued mappings and $f, g, p: C L(X, Y) \longrightarrow C L(X, Y)$ be single-valued mappings. Suppose the maps $\eta: K \times K \longrightarrow X$ and $h: K \times$ $K \longrightarrow Y$ are two bifunctions.

Problem 1. Suppose $C \subseteq Y$ is a closed, convex, pointed cone with int $C \neq \varnothing$. Then, the generalized nonlinear vector variational-like inequality problem (I) (GNVVLIP (I)) is to find $x_{0} \in K$, such that for each $y \in K$, there exist $u \in M\left(x_{0}\right)$, $v \in S\left(x_{0}\right)$, and $w \in T\left(x_{0}\right)$ such that

$$
((p(u)-(f(v)-g(w))))\left(\eta\left(y, x_{0}\right)\right)-h\left(x_{0}, y\right) \notin-\operatorname{int} C .
$$

Problem 2. Suppose $C: K \longrightarrow Y$ is a set-valued map such that for every $x \in K, C(x)$ is a proper, closed, convex, pointed cone with nonempty interior. Then, the generalized nonlinear vector variational-like inequality problem (II) (GNVVLI (II)) is to find $x_{0} \in K$ such that for each $y \in K, \exists u \in M\left(x_{0}\right)$, $v \in S\left(x_{0}\right)$, and $w \in T\left(x_{0}\right)$ such that

$$
((p(u)-(f(v)-g(w))))\left(\eta\left(y, x_{0}\right)\right)-h\left(x_{0}, y\right) \notin-\operatorname{int} C\left(x_{0}\right) .
$$

Here, we are trying to provide solutions to the above stated generalized nonlinear vector variational-like inequality problems (I and II) from a topological point of view. We consider $X$ and $Y$ to be any topological vector spaces and use the concept of admissibility of the function space topology along with net theory to prove the existence of solutions of these generalized nonlinear vector variational-like inequality problems.

\section{Preliminaries}

Below, we provide some definitions and results related mainly to set-valued maps between topological spaces.

Definition 3 [23]. Suppose $\left(X, \tau_{1}\right)$ and $\left(Y, \tau_{2}\right)$ are two topological spaces and $F: X \longrightarrow Y$ is a set-valued map.

(i) $F$ is called upper semicontinuous (in short, u.s.c.) at a point $x \in X$, if for every open set $V$ in $Y$ such that $F(x) \subseteq V$, there exists an open set $U$ in $X$ with $x \in$ $U$ such that $F(U) \subseteq V$;

(ii) $F$ is called lower semicontinuous (in short, l.s.c.) at a point $x \in X$, if for every open set $V$ in $Y$ such that $F(x) \cap V \neq \varnothing$, there exists an open set $U$ in $X$ with $x \in U$ such that for each $u \in U, F(u) \cap V \neq \varnothing$;

(iii) $F$ is said to be continuous at $x \in X$ if it is both upper semicontinuous and lower semicontinuous at $x$;

(iv) $F$ is said to be continuous (resp. u.s.c. and l.s.c.) if it is so at each point of $X$.

Lemma 4 [24]. Suppose $\left(X, \tau_{1}\right)$ and $\left(Y, \tau_{2}\right)$ are topological spaces and $F: X \longrightarrow Y$ is a set-valued map. Then,

(i) if $F$ is upper semicontinuous at $x \in X$ and $F(x)$ is compact then for every net $\left\{x_{\alpha}\right\}_{\alpha \in D}$ and $y_{\alpha} \in F\left(x_{\alpha}\right)$ with $x_{\alpha} \longrightarrow x$ and $y_{\alpha} \longrightarrow y$, we have $y \in F(x)$;

(ii) $F$ is lower semicontinuous at $x \in X$ if and only if for every $y \in F(x)$ and every net $\left\{x_{\alpha}\right\}_{\alpha \in D}$ with $x_{\alpha} \longrightarrow x$, there exists a subnet $\left\{x_{\beta}\right\}_{\beta \in \Omega}$, where $\Omega$ is a directed subset of $D$ and a net $\left\{y_{\beta}\right\}_{\beta \in \Omega}$ such that $y_{\beta} \in F\left(x_{\beta}\right)$ with $y_{\beta} \longrightarrow y$.

Theorem 5 [23]. Let $(X, \tau)$ and $(Y, \mu)$ be two topological spaces. Let $F: X \longrightarrow Y$ be a set-valued map. Then, $F$ is lower semicontinuous at $x \in X$ if for any net $\left\{x_{n}\right\}_{n \in \Delta}$ in $X$ converging to $x \in X$, the image net $\left\{F\left(x_{n}\right)\right\}_{n \in \Delta}$ converges to $F(x)$.

Lemma 6 [25]. Suppose $X$ and $Y$ are topological spaces and $F: X \longrightarrow Y$ is a set-valued upper semicontinuous function. If $F(x)$ is compact for each $x \in X$, then image of every compact subset of $X$ under $F$ is compact.

Definition $7[23,26]$. Let $\left(Y, \mu_{1}\right)$ and $\left(Z, \mu_{2}\right)$ be two topological spaces. Let $\mathscr{C}(Y, Z)$ be the space of all continuous mappings from $Y$ to $Z$. A topology $\tau$ on $\mathscr{C}(Y, Z)$ is called admissible, if the evaluation map $e: \mathscr{C}(Y, Z) \times Y \longrightarrow Z$, defined by $e(f, y)=f(y)$, is continuous.

Definition 8 [26]. Let $\left\{f_{n}\right\}_{n \in \Delta}$ be a net in $\mathscr{C}(Y, Z)$. Then, $\left\{f_{n}\right\}_{n \in \Delta}$ is said to continuously converge to $f$ if for each net $\left\{y_{m}\right\}_{m \in \sigma}$ in $Y$ converging to $y,\left\{f_{n}\left(y_{m}\right)\right\}_{(n, m) \in \Delta \times \sigma}$ converges to $f(y)$ in $Z$. 
Theorem 9 [23]. Let $(Y, \tau)$ and $(Z, \mu)$ be two topological spaces. A topology $\mathfrak{T}$ on $\mathscr{C}(Y, Z)$, the family of continuous mappings from $Y$ to $Z$, is admissible if and only if for any net $\left\{f_{n}\right\}_{n \in \Delta}$ in $\mathscr{C}(Y, Z),\left\{f_{n}\right\}_{n \in \Delta}$ converges to $f$ in $\mathfrak{I}$ implies continuous convergence of $\left\{f_{n}\right\}_{n \in \Delta}$ to $f$.

Definition 10 [27]. Suppose $F: X \longrightarrow Y$ is a set-valued map from $X$ to $Y$. The graph of $F$, denoted by $\mathscr{G}(F)$, is

$$
\mathscr{G}(F)=\{(x, y) \in X \times Y \mid x \in X, y \in F(x)\}
$$

Definition 11 [28]. Suppose $S$ is a nonempty subset of some topological vector space $X$. A set-valued map $F: S \longrightarrow X$ is called a KKM-mapping if for every nonempty finite set $\left\{x_{1}\right.$, $\left.x_{2}, \cdots, x_{n}\right\}$ of $S$, we have

$$
\operatorname{conv}\left\{x_{1}, x_{2}, \cdots, x_{n}\right\} \subseteq \bigcup_{j=1}^{n} F\left(x_{j}\right)
$$

The following result is taken from [28].

Lemma 12 (KKM-Theorem). Suppose $S$ is a nonempty subset of some topological vector space $X$ and $F: S \longrightarrow X$ is a KKMmapping such that for every $x \in S, F(x)$ is a closed subset of $X$. If there exists a point $x_{0} \in S$ such that $F\left(x_{0}\right)$ is compact, then $\bigcap_{x \in S} F(x) \neq \varnothing$.

\section{Main Results}

Theorem 13. Suppose $X$ and $Y$ are two topological vector spaces and $C L(X, Y)$ is the space of all continuous linear mappings from the space $X$ to the space $Y$ equipped with an admissible topology. Let $K$ be a nonempty, compact, closed, and convex subset of $X$. Suppose $C \subseteq Y$ is a closed, convex, pointed cone with int $C \neq \varnothing$. Further, let $M, S, T: K \longrightarrow C L(X, Y)$ be set-valued lower semicontinuous mappings and $f, g, p: C L$ $(X, Y) \longrightarrow C L(X, Y)$ be continuous mappings. Suppose the maps $\eta: K \times K \longrightarrow X$ and $h: K \times K \longrightarrow Y$ are affine mappings such that $\eta$ is continuous in the second argument and $h$ is continuous in the first argument, respectively, with $\eta(x, x)=h(x, x)=0$ for all $x \in K$. Then, the generalized nonlinear vector variational-like inequality problem (I) has a solution. That is, there exists $x_{0} \in K$ such that for each $y \in K$, there exist $u \in M\left(x_{0}\right), v \in S\left(x_{0}\right)$, and $w \in T\left(x_{0}\right)$ such that

$$
((p(u)-(f(v)-g(w))))\left(\eta\left(y, x_{0}\right)\right)-h\left(x_{0}, y\right) \notin-\text { int } C .
$$

Proof. We define a set-valued map $F: K \longrightarrow K$ by

$$
\begin{aligned}
F(y)= & \{x \in K \mid \exists u \in M(x), v \in S(x), w \in T(x) \text { such that } \\
& \cdot((p(u)-(f(v)-g(w))))(\eta(y, x))-h(x, y) \notin-i n t C\} .
\end{aligned}
$$

Clearly, $F(y)$ is nonempty as $y \in F(y)$. As $y \in K$, we have $\eta(y, y)=h(y, y)=0$. Thus, for each $u \in M(y), v \in S(y)$, and $w \in T(x)$, we have $(p(u)-(f(v)-g(w)))(\eta(y, y))-h(y, y)$ $=(p(u)-(f(v)-g(w)))(0)-0=\mathbf{0}$. Since $C$ is a closed convex and pointed cone, thus $\mathbf{0} \notin-$ int $C$.

The proof of the theorem is divided into two parts:

(i) $F$ is a KKM-mapping on $K$ :

Let $A=\left\{x_{1}, x_{2}, \cdots, x_{n}\right\} \subseteq K$ be any finite subset of $K$.

We show that $\operatorname{conv}\left\{x_{1}, x_{2}, \cdots, x_{n}\right\} \subseteq \bigcup_{i=1}^{n} F\left(x_{i}\right)$. Let, if possible, $x^{\prime} \notin \bigcup_{i=1}^{n} F\left(x_{i}\right)$ for some $x^{\prime} \in \operatorname{conv}\left\{x_{1}, x_{2}, \cdots, x_{n}\right\}$. Then, we have $x^{\prime}=\sum_{i=1}^{n} \mu_{i} x_{i}$ for some $\mu_{i} \geq 0$ and $\sum_{i=1}^{n} \mu_{i}=1$. Also, as $x^{\prime} \notin F\left(x_{i}\right)$, for all $u \in M\left(x^{\prime}\right), \quad v \in S\left(x^{\prime}\right)$, and $w \in T\left(x^{\prime}\right)$, we have $((p(u)-(f(v)-g(w))))\left(\eta\left(x_{i}, x^{\prime}\right)\right)-h($ $\left.x^{\prime}, x_{i}\right) \in-$ int $C$, for each $i=1,2, \cdots, n$. Since -int $C$ is convex and $\mu_{i} \geq 0$ with $\sum_{i=1}^{n} \mu_{i}=1$, therefore $\sum_{i=1}^{n} \mu_{i}[(p(u)-(f(v)-$ $\left.g(w)))\left(\eta\left(x_{i}, x^{\prime}\right)\right)-h\left(x^{\prime}, x_{i}\right)\right] \in-$ int $C$. As $p(u), f(v)$, and $g(w)$ belong to $C L(X, Y)$, they are linear. Therefore, we have $\sum_{i=1}^{n} \mu_{i}\left[(p(u)-(f(v)-g(w)))\left(\eta\left(x_{i}, x^{\prime}\right)\right)-h\left(x^{\prime}, x_{i}\right)\right]=(p(u)$ $-(f(v)-g(w)))\left(\sum_{i=1}^{n} \mu_{i} \eta\left(x_{i}, x^{\prime}\right)\right)-\sum_{i=1}^{n} \mu_{i} h\left(x^{\prime}, x_{i}\right)$. Again, $\eta$ and $h$ are affine; hence, $\sum_{i=1}^{n} \mu_{i}\left(\eta\left(x_{i}, x^{\prime}\right)\right)-\sum_{i=1}^{n} \mu_{i}\left(h\left(x^{\prime}, x_{i}\right)\right)$ $=\eta\left(\sum_{i=1}^{n} \mu_{i} x_{i}, \sum_{i=1}^{n} \mu_{i} x^{\prime}\right)-h\left(\sum_{i=1}^{n} \mu_{i} x^{\prime}, \sum_{i=1}^{n} \mu_{i} x_{i}\right)=\eta\left(x^{\prime}, x^{\prime}\right)-$ $h\left(x^{\prime}, x^{\prime}\right)=\mathbf{0}$ as $\eta\left(x^{\prime}, x^{\prime}\right)=\mathbf{0}=h\left(x^{\prime}, x^{\prime}\right)$ by the given hypothesis. Therefore, $\sum_{i=1}^{n} \mu_{i}\left[p(u)-(f(v)-g(w))\left(\eta\left(x_{i}, x^{\prime}\right)\right)-h\left(x^{\prime}\right.\right.$ ,$\left.\left.x_{i}\right)\right]=\mathbf{0}$. Thus, we have $\mathbf{0} \in-$ int $C$, where $\mathbf{0}$ is the zero vector in $Y$. Thus, $\mathbf{0}=\mathbf{- 0} \in$ int $C$, which is a contradiction. Therefore, we have $\operatorname{conv}\left\{x_{1}, x_{2}, \cdots, x_{n}\right\} \subseteq \bigcup_{i=1}^{n} F\left(x_{i}\right)$. Hence, $F$ is a KKMmapping on $K$.

(ii) $F(y)$ is closed for each $y \in K$ :

Let $\left\{x_{\alpha}\right\}_{\alpha \in D}$ be a net in $F(y)$, converging to some $z_{0}$ in $X$. As $K$ is closed, $z_{0} \in K$. We have to show that $z_{0} \in F(y)$, that is, there exist $u_{0} \in M\left(z_{0}\right), v_{0} \in S\left(z_{0}\right)$, and $w_{0} \in T\left(z_{0}\right)$ such that $\left(p\left(u_{0}\right)-\left(f\left(v_{0}\right)-g\left(w_{0}\right)\right)\right)\left(\eta\left(y, z_{0}\right)\right)-h\left(z_{0}, y\right) \notin-$ int $C$. Since $x_{\alpha} \in F(y)$, therefore there exist some $u_{\alpha} \in M\left(x_{\alpha}\right), v_{\alpha} \in S\left(x_{\alpha}\right)$, and $w_{\alpha} \in T\left(x_{\alpha}\right)$ such that $\left(p\left(u_{\alpha}\right)-\left(f\left(v_{\alpha}\right)-g\left(w_{\alpha}\right)\right)\right)\left(\eta\left(y, x_{\alpha}\right.\right.$ )$)-h\left(x_{\alpha}, y\right) \notin-$ int $C$. Now, the maps $M, S$, and $T$ are setvalued lower semicontinuous functions; therefore, for each $u_{0} \in M\left(z_{0}\right)$ and $\left\{x_{\alpha}\right\}$ converging to $z_{0}$, there exists a subnet $\left\{x_{\alpha_{k}}\right\}$ of $\left\{x_{\alpha}\right\}$ with $u_{\alpha_{k}} \in M\left(x_{\alpha_{k}}\right)$ such that $u_{\alpha_{k}}$ converges to $u_{0}$, in view of Lemma 4 . Now, $\left\{x_{\alpha_{k}}\right\}$ is a net in itself converging to $z_{0}$ and $w_{0} \in T\left(z_{0}\right)$; therefore, there exists a subnet $\left\{x_{\alpha_{k_{l}}}\right\}$ of $\left\{x_{\alpha_{k}}\right\}$ with $v_{\alpha_{k_{l}}} \in S\left(x_{\alpha_{k_{l}}}\right)$. Similarly, we have a subnet $\left\{x_{\alpha_{k_{l m}}}\right\}$ of $\left\{x_{\alpha_{k_{l}}}\right\}$ with $w_{\alpha_{k_{l m}}} \in T\left(x_{\alpha_{k_{l m}}}\right)$ such that the subnets $\left\{u_{\alpha_{k}}\right\},\left\{v_{\alpha_{k_{l}}}\right\}$, and $\left\{w_{\alpha_{k_{l m}}}\right\}$ converge to $u_{0}, v_{0}$, and $w_{0}$, respectively, in view of Lemma 4 . As these nets are subnets of $u_{\alpha_{k}}$, $v_{\alpha_{k}}$, and $w_{\alpha_{k}}$, respectively, thus without loss of generality, we denote the subnets $\left\{u_{\alpha_{k}}\right\},\left\{v_{\alpha_{k_{l}}}\right\}$, and $\left\{w_{\alpha_{k_{l m}}}\right\}$ by $\left\{u_{\alpha_{k}}\right\}$, $\left\{v_{\alpha_{k}}\right\}$, and $\left\{w_{\alpha_{k}}\right\}$, respectively, which converge to $u_{0}, v_{0}$, and $w_{0}$, respectively.

Since the single-valued map $p, f$, and $g$ are continuous, therefore we have $p\left(u_{\alpha_{k}}\right), f\left(v_{\alpha_{k}}\right)$, and $g\left(w_{\alpha_{k}}\right)$ converge to $p\left(u_{0}\right), f\left(v_{0}\right)$, and $g\left(w_{0}\right)$, respectively.

By the given hypothesis, that is, $\eta$ is continuous in the second argument, we have $\eta\left(y, x_{\alpha_{k}}\right)$ converges to $\eta\left(y, z_{0}\right)$. Since the space $C L(X, Y)$ is given to be admissible, thus we 
have $\left(p\left(u_{\alpha_{k}}\right)-\left(f\left(v_{\alpha_{k}}\right)-g\left(w_{\alpha_{k}}\right)\right)\right)\left(\eta\left(y, x_{\alpha_{k}}\right)\right)$ converges to $\left(p\left(u_{0}\right)-\left(f\left(v_{0}\right)-g\left(w_{0}\right)\right)\right)\left(\eta\left(y, z_{0}\right)\right)$, by Theorem 9. As the map $h$ is continuous in the first component, therefore $h\left(x_{\alpha_{k}}, y\right)$ converges to $h\left(z_{0}, y\right)$. Hence, $\left(\left(p\left(u_{\alpha_{k}}\right)-\left(f\left(v_{\alpha_{k}}\right)-g\right.\right.\right.$ $\left.\left.\left.\left(w_{\alpha_{k}}\right)\right)\right)\left(\eta\left(y, x_{\alpha_{k}}\right)\right)-h\left(x_{\alpha_{k}}, y\right)\right)$ converges to $\left(p\left(u_{0}\right)-\left(f\left(v_{0}\right)\right.\right.$ $\left.\left.-g\left(w_{0}\right)\right)\right)\left(\eta\left(y, z_{0}\right)\right)-h\left(z_{0}, y\right)$, in view of the fact that $C L(X$, $Y)$ is admissible.

Now, we will show that $\left(p\left(u_{0}\right)-\left(f\left(v_{0}\right)-g\left(w_{0}\right)\right)\right)(\eta(y$, $\left.\left.z_{0}\right)\right)-h\left(z_{0}, y\right) \notin$-int $C$.

Let, if possible, $\left(p\left(u_{0}\right)-\left(f\left(v_{0}\right)-g\left(w_{0}\right)\right)\right)\left(\eta\left(y, z_{0}\right)\right)-h($ $\left.z_{0}, y\right) \in-$ int $C$. Then, by the convergence of net, we have ( $p$ $\left.\left(u_{\alpha_{k}}\right)-\left(f\left(v_{\alpha_{k}}\right)-g\left(w_{\alpha_{k}}\right)\right)\right)\left(\eta\left(y, x_{\alpha_{k}}\right)\right)-h\left(x_{\alpha_{k}}, y\right) \in-\operatorname{int} C$ eventually, which leads to contradiction. Hence, $\left(p\left(u_{0}\right)-(f\right.$ $\left.\left.\left(v_{0}\right)-g\left(w_{0}\right)\right)\right)\left(\eta\left(y, z_{0}\right)\right)-h\left(z_{0}, y\right) \notin-$ int $C$. Thus, we have $z_{0} \in F(y)$.

Now $F(y)$ is closed, and $K$ is compact. This implies that $F(y)$ is a compact subset of $K$. Therefore, by KKM-Theorem, $\bigcap_{y \in K} F(y) \neq \varnothing$. Hence, there exists some $x_{0} \in K$ such that $x_{0}$ $\in \bigcap_{y \in K} F(y)$. That is, for each $y \in K$, there exist $u_{0} \in M\left(x_{0}\right)$, $v_{0} \in S\left(x_{0}\right)$, and $w_{0} \in T\left(x_{0}\right)$ such that $\left(p\left(u_{0}\right)-\left(f\left(v_{0}\right)-g(\right.\right.$ $\left.\left.w_{0}\right)\right)\left(\eta\left(y, x_{0}\right)\right)-h\left(x_{0}, y\right) \notin-$ int $C$, hence the result.

In the above theorem, we have proved that, along with other conditions, lower semicontinuity of $M, S, T$ ensures existence of solutions for GNVVLIP (I).

In the next theorem, we are providing another set of conditions for the existence of solutions for these class of problems.

Theorem 14. Suppose $X$ and $Y$ are two topological vector spaces and $C L(X, Y)$ is the space of all continuous linear mappings from the space $X$ to the space $Y$ equipped with an admissible topology. Let $K$ be a nonempty, closed, compact, and convex subset of $X$. Suppose $C \subseteq Y$ is a closed, convex, pointed cone with int $C \neq \varnothing$. Further, let $M, S, T: K \longrightarrow C L(X, Y)$ be set-valued upper semicontinuous functions with nonempty compact values, that is, $M(x), S(x)$, and $T(x)$ are compact for every $x \in K$. Let $f, g, p: C L(X, Y) \longrightarrow C L(X, Y)$ be continuous mappings. Suppose the maps $\eta: K \times K \longrightarrow X$ and $h: K \times K \longrightarrow Y$ are affine mappings such that $\eta$ is continuous in the second argument and $h$ is continuous in the first argument, respectively, with $\eta(x, x)=h(x, x)=0$ for all $x \in K$. Then, there exists a solution to the generalized nonlinear vector variational inequality problem. That is, there exists $x_{0} \in K$ such that for each $y \in K$, there exist $u \in M\left(x_{0}\right), v \in S\left(x_{0}\right)$, and $w \in T\left(x_{0}\right)$ such that

$$
((p(u)-(f(v)-g(w))))\left(\eta\left(y, x_{0}\right)\right)-h\left(x_{0}, y\right) \notin-\operatorname{int} C .
$$

Proof. Consider a set-valued map $F: K \longrightarrow K$ defined as

$$
\begin{aligned}
F(y)= & \{x \in K \mid \exists u \in M(x), v \in S(x), w \in T(x) \text { such that } \\
& \cdot((p(u)-(f(v)-g(w))))(\eta(y, x))-h(x, y) \notin-i n t C\} .
\end{aligned}
$$

The proof of the theorem is divided into two parts:

(i) $F$ is a KKM-mapping on $K$; (ii) $F(y)$ is closed for each $y \in K$.

Proof of part (i) is similar to that of Theorem 13. Therefore, we are providing the proof of part (ii) only.

Let $\left\{x_{\alpha}\right\}_{\alpha \in D}$ be a net in $F(y)$, converging to some $z_{0} \in X$. As $K$ is closed, $z_{0} \in K$. We have to show that $z_{0} \in F(y)$, that is, there exist $u_{0} \in M\left(z_{0}\right), v_{0} \in S\left(z_{0}\right)$, and $w_{0} \in T\left(z_{0}\right)$ such that $\left(p\left(u_{0}\right)-\left(f\left(v_{0}\right)-g\left(w_{0}\right)\right)\right)\left(\eta\left(y, z_{0}\right)\right)-h\left(z_{0}, y\right) \notin-$ int $C$. Since $x_{\alpha} \in F(y)$, therefore there exist some $u_{\alpha} \in M\left(x_{\alpha}\right), w_{\alpha} \in T\left(x_{\alpha}\right)$, and $v_{\alpha} \in S\left(x_{\alpha}\right)$ such that $\left(p\left(u_{\alpha}\right)-\left(f\left(v_{\alpha}\right)-g\left(w_{\alpha}\right)\right)\right)\left(\eta\left(y, x_{\alpha}\right)\right)$ $-h\left(x_{\alpha}, y\right) \notin$-int $C$. Now, $K$ is a compact subset of $X$, and $M$ ,$S$, and $T$ are set-valued upper semicontinuous functions such that $M(x), S(x)$, and $T(x)$ are compact. Therefore, $M(K)$, $S(K)$, and $T(K)$ are also compact by Lemma 6 . As $\left\{u_{\alpha}\right\}$, $\left\{v_{\alpha}\right\}$, and $\left\{w_{\alpha}\right\}$ are nets in $M(K), S(K)$, and $T(K)$, respectively, there exist subnets $\left\{u_{\alpha_{k}}\right\}_{\alpha_{k} \in D_{1}}, \quad\left\{v_{\alpha_{l}}\right\}_{\alpha_{1} \in D_{2}}$, and $\left\{w_{\alpha_{m}}\right\}_{\alpha_{m} \in D_{3}}$ such that $\left\{u_{\alpha_{k}}\right\},\left\{v_{\alpha_{l}}\right\}$, and $\left\{w_{\alpha_{m}}\right\}$ converge to some $u_{0} \in M(K), v_{0} \in S(K)$, and $w_{0} \in T(K)$, respectively.

Now, we construct a directed set $D_{4} \subset D$, defined in the following way:

By the order property of the directed set $D$, for each triplet $\alpha_{k}, \alpha_{l}, \alpha_{m} \in D$, there exists some $\alpha_{\delta} \in D$ such that $\alpha_{\delta} \geq \alpha_{k}$, $\alpha_{\delta} \geq \alpha_{l}$, and $\alpha_{\delta} \geq \alpha_{m}$. We denote the collection of such $\alpha_{\delta}$ s by $D_{4}$. It can be easily verified that $D_{4}$ is a directed set under the induced ordering of $D$.

Thus, we have subnets $\left\{u_{\delta}\right\}_{\delta \in D_{4}},\left\{v_{\delta}\right\}_{\delta \in D_{4}}$, and $\left\{w_{\delta}\right\}_{\delta \in D_{4}}$ of $\left\{u_{\alpha_{k}}\right\},\left\{v_{\alpha_{l}}\right\}$, and $\left\{w_{\alpha_{m}}\right\}$, respectively, such that $\left\{u_{\delta}\right\},\left\{v_{\delta}\right\}$, and $\left\{w_{\delta}\right\}$ converge to $u_{0} \in M(K), v_{0} \in S(K)$, and $w_{0} \in T(K)$, respectively.

Then, proceeding as in Theorem 13, we have $z_{0} \in F(y)$. Thus, $F(y)$ is closed, and $K$ is compact. This implies that $F$ $(y)$ is a compact subset of $K$. Therefore, by KKM-Theorem, there exists some $x_{0} \in F(y)$ for each $y \in K$. That is, for each $y \in K$, there exist $u_{0} \in M\left(x_{0}\right), v_{0} \in S\left(x_{0}\right)$, and $w_{0} \in T\left(x_{0}\right)$ such that $\left(p\left(u_{0}\right)-\left(f\left(v_{0}\right)-g\left(w_{0}\right)\right)\right)\left(\eta\left(y, x_{0}\right)\right)-h\left(x_{0}, y\right) \notin-$ int $C$, hence the result.

In the next theorem, we investigate the properties of solution sets of GNVVLIP (I).

Theorem 15. Let $S \subseteq K$ be the set of all solutions of a generalized nonlinear vector variational-like inequality problem as obtained in Theorem 13 (respectively, Theorem 14). Then, $S$ is closed and compact in $K$.

Proof. Suppose $S \subseteq K$ is the solution set of the generalized nonlinear vector variational-like inequality problem. Then, by Theorem 13, we have $S=\bigcap_{y \in K} F(y)$, where

$$
\begin{aligned}
F(y)= & \{x \in K \mid \exists u \in M(x), v \in S(x), w \in T(x) \text { such that } \\
& \cdot((p(u)-(f(v)-g(w))))(\eta(y, x))-h(x, y) \notin-i n t C\} .
\end{aligned}
$$

It has been proved in Theorem 13 that each $F(y)$ is closed. Therefore, $S$ is a closed set and hence a closed subset of $K$. Since $K$ is compact, $S$ is compact as well. 
In the next set of theorems, we consider the other variant of the generalized nonlinear vector variational-like inequality problem and provide the conditions for the solution.

Theorem 16. Suppose $X$ and $Y$ are two topological vector spaces and $C L(X, Y)$ is the space of all continuous linear mappings from the space $X$ to the space $Y$ equipped with an admissible topology. Let $K$ be a nonempty, closed, compact, and convex subset of $X$. Suppose $C: K \longrightarrow Y$ is a set-valued map such that for every $x \in K, C(x)$ is a proper closed, convex, pointed cone with int $C(x) \neq \varnothing$. Further, suppose that $G: K$ $\longrightarrow Y$ is also a set-valued map defined by $G(x)=Y \backslash(-$ int $C(x))$ such that the graph of $G, \mathscr{G}(G)$, is a closed set in $X \times$ $Y$. Further, let $M, S, T: K \longrightarrow C L(X, Y)$ be set-valued lower semicontinuous mappings and $f, g, p: C L(X, Y) \longrightarrow C L(X$, $Y)$ be continuous mappings. Suppose the maps $\eta: K \times K$ $\longrightarrow X$ and $h: K \times K \longrightarrow Y$ are affine mappings such that $\eta$ is continuous in the second argument and $h$ is continuous in the first argument, respectively, with $\eta(x, x)=h(x, x)=0$ for all $x \in K$. Then, the generalized nonlinear vector variationallike inequality problem (II) has a solution. That is, there exists $x_{0} \in K$ such that for each $y \in K, \exists u \in M\left(x_{0}\right), v \in S\left(x_{0}\right)$, and $w \in T\left(x_{0}\right)$ such that

$$
((p(u)-(f(v)-g(w))))\left(\eta\left(y, x_{0}\right)\right)-h\left(x_{0}, y\right) \notin-\operatorname{int} C\left(x_{0}\right) .
$$

Proof. Consider a set-valued map $F: K \longrightarrow K$ defined as

$$
\begin{aligned}
F(y)= & \{x \in K \mid \exists u \in M(x), v \in S(x), w \in T(x) \text { such that } \\
& \left.\cdot((p(u)-(f(v)-g(w))))(\eta(y, x))-h(x, y) \notin-\operatorname{int} C\left(x_{0}\right)\right\} .
\end{aligned}
$$

Likewise in Theorem 13, the proof of this theorem is also divided into two parts:

\section{(i) $F$ is a KKM-mapping on $K$;}

(ii) $F(y)$ is closed for each $y \in K$.

Proof of part (i) is similar to that of Theorem 13. Therefore, we are providing the proof of part (ii) only.

As in Theorem 13, it follows that the net $\left\{\left(p\left(u_{\alpha_{k}}\right)-(f(\right.\right.$ $\left.\left.\left.\left.v_{\alpha_{k}}\right)-g\left(w_{\alpha_{k}}\right)\right)\right)\left(\eta\left(y, x_{\alpha_{k}}\right)\right)-h\left(x_{\alpha_{k}}, y\right)\right\}$ converges to $\left(p\left(u_{0}\right)-\right.$ $\left.\left(f\left(v_{0}\right)-g\left(w_{0}\right)\right)\right)\left(\eta\left(y, z_{0}\right)\right)-h\left(z_{0}, y\right)$, in view of admissibility of $C L(X, Y)$, lower semicontinuity of $T$, and the other given conditions of the hypothesis.

Now, we will show that $\left(p\left(u_{0}\right)-\left(f\left(v_{0}\right)-g\left(w_{0}\right)\right)\right)(\eta(y$, $\left.\left.z_{0}\right)\right)-h\left(z_{0}, y\right) \notin-$ int $C\left(z_{0}\right)$. As in Theorem 13, we have the net $\left\{x_{\alpha},\left(p\left(u_{\alpha_{k}}\right)-\left(f\left(v_{\alpha_{k}}\right)-g\left(w_{\alpha_{k}}\right)\right)\right)\left(\eta\left(y, x_{\alpha_{k}}\right)\right)-h\left(x_{\alpha_{k}}, y\right)\right\}$ is convergent and converges to $\left\{z_{0},\left(p\left(u_{0}\right)-\left(f\left(v_{0}\right)-g(\right.\right.\right.$ $\left.\left.\left.w_{0}\right)\right)\left(\eta\left(y, z_{0}\right)\right)-h\left(z_{0}, y\right)\right\}$. Also, the net is contained in $\mathscr{G}(G)$, and the graph $\mathscr{G}(G)$ is closed; therefore, $\left(z_{0},\left(p\left(u_{0}\right)\right.\right.$ $\left.\left.-\left(f\left(v_{0}\right)-g\left(w_{0}\right)\right)\right)\left(\eta\left(y, z_{0}\right)\right)-h\left(z_{0}, y\right)\right) \in \mathscr{G}(G)$. Hence, we have $\left.p\left(u_{0}\right)-\left(f\left(v_{0}\right)-g\left(w_{0}\right)\right)\right)\left(\eta\left(y, z_{0}\right)\right)-h\left(z_{0}, y\right) \notin-$ int $C($ $\left.z_{0}\right)$. Thus, we have $z_{0} \in F(y)$. Therefore, $F(y)$ is closed.
Now $F(y)$ is closed, and $K$ is compact. This implies that $F(y)$ is a compact subset of $K$. Therefore, by KKM-Theorem, $\bigcap_{y \in K} F(y) \neq \varnothing$. Hence, there exists some $x_{0} \in K$ such that $x_{0}$ $\in \bigcap_{y \in K} F(y)$. That is, for each $y \in K$, there exist $u_{0} \in M\left(x_{0}\right)$, $v_{0} \in S\left(x_{0}\right)$, and $w_{0} \in T\left(x_{0}\right)$ such that $\left(p\left(u_{0}\right)-\left(f\left(v_{0}\right)-g(\right.\right.$ $\left.\left.\left.w_{0}\right)\right)\right)\left(\eta\left(y, x_{0}\right)\right)-h\left(x_{0}, y\right) \notin-$ int $C\left(x_{0}\right)$, hence the result.

Another solution of the generalized nonlinear vector variational-like inequality problem (II) is provided below.

Theorem 17. Suppose $X$ and $Y$ are two topological vector spaces and $C L(X, Y)$ is the space of all continuous linear mappings from the space $X$ to the space $Y$ equipped with an admissible topology. Let $K$ be a nonempty, closed, compact, and convex subset of $X$. Suppose $C: K \longrightarrow Y$ is a set-valued map such that for every $x \in K, C(x)$ is a proper, closed, convex, pointed cone with int $C(x) \neq \varnothing$. Further, suppose that $G: K$ $\longrightarrow Y$ is also a set-valued map defined by $G(x)=Y \backslash(-$ int $C(x))$ such that graph of $G, \mathscr{G}(G)$, is a closed set in $X \times Y$. Further, let $M, S, T: K \longrightarrow C L(X, Y)$ be set-valued upper semicontinuous functions with nonempty compact values, that is, $M(x), S(x)$, and $T(x)$ are compact for every $x \in K$ and let $f$, $g, p: C L(X, Y) \longrightarrow C L(X, Y)$ be continuous mappings. Suppose the maps $\eta: K \times K \longrightarrow X$ and $h: K \times K \longrightarrow Y$ are affine mappings such that $\eta$ is continuous in the second argument and $h$ is continuous in the first argument with $\eta(x, x)$ $=h(x, x)=0$, respectively, for all $x \in K$. Then, the generalized nonlinear vector variational-like inequality problem (II) has a solution. That is, there exists $x_{0} \in K$ such that for each $y \in K$, $\exists u \in M\left(x_{0}\right), v \in S\left(x_{0}\right)$, and $w \in T\left(x_{0}\right)$ such that

$$
((p(u)-(f(v)-g(w))))\left(\eta\left(y, x_{0}\right)\right)-h\left(x_{0}, y\right) \notin-\operatorname{int} C\left(x_{0}\right) .
$$

Proof. The result can be proved on the similar lines as that of Theorem 14 and Theorem 16.

We can draw the following conclusions from the results obtained so far:

(i) Suppose $h: K \times K \longrightarrow Y$ is defined as the constant map with $h(x, y)=0$ for all $(x, y) \in K \times K$ and $\eta: K$ $\times K \longrightarrow Y$ is defined as $\eta(x, y)=x-y$, and $M$ and $S$ are taken as zero functions. If $f, p: C L(X, Y) \longrightarrow C L$ $(X, Y)$ are also defined as zero functions, that if $f(x)$ $=p(x)=0$ for all $x \in C L(X, Y)$ and $g: C L(X, Y)$ $\longrightarrow C L(X, Y)$ is taken as an identity map, that is, $g(x)=x$ for all $x \in C L(X, Y)$. Then, Theorem 16 ensures solution to the generalized vector variational inequality problem discussed in [29]. Also, we have

(a) Theorem 16 reduces to Theorem 3.6 of [29];

(b) Theorem 17 reduces to Theorem 3.1 of [29].

Further, if $T$ is single-valued, then Theorem 13 reduces to Theorem 3.1 and Theorem 17 reduces to Theorem 3.2 of [30], respectively. 


\section{Conclusion}

In this paper, we have provided solutions to two variants of the generalized vector variational-like inequality problem. Our approach and the result obtained here differ significantly from those of the existing literature. To be precise,

(i) The spaces $X$ and $Y$ considered in this paper are topological vector spaces. In [15], the space $Y$ is taken to be $\mathbb{R}$, whereas [13] deals with real Hilbert spaces;

(ii) We have used conditions of upper semicontinuity as well as lower semicontinuity on the set-valued mappings to obtain our results. In [15], the concepts of transfer closed and intersectionally closed are used along with an assumption milder than $C$-pseudomonotonicity on the set-valued mappings. On the other hand, relaxed monotonicity and relaxed Lipschitz's continuity are used in [13] and generalized $C$-quasi-convexity is used in [17] for the setvalued mappings.

The approach adopted in our paper is topological and varies significantly from the rest literature. Net theory is extensively used in all the main results. We have used admissibility of the function space topology to obtain our results. The authors are not aware of any such results in the literature of variational inequality which are proved adopting similar techniques.

\section{Data Availability}

No data is used.

\section{Conflicts of Interest}

The authors declare there is no conflict of interest.

\section{Acknowledgments}

The authors thank the referees for their valuable suggestions. The authors also thank Professor C. S. Lalitha for her valuable suggestions and remarks which helped improve the paper.

\section{References}

[1] R. P. Agarwal, Salahuddin, and M. K. Ahmad, "Hybrid-type generalized multivalued vector complementarity problems," Ukrainian Mathematical Journal, vol. 65, no. 1, pp. 5-20, 2013.

[2] S. S. Chang, Fixed Point Theory with Applications, Chongquing Publishing House, Chongqing, 1984.

[3] S. Dafermos, "Traffic equilibrium and variational inequalities," Transportation Science, vol. 14, no. 1, pp. 42-54, 1980.

[4] F. Ferro, "A minimax theorem for vector valued functions," Journal of Optimization Theory and Applications, vol. 60, no. 1, pp. 19-31, 1989.

[5] F. E. Browder, "Existence and approximation of solutions of nonlinear variational inequalities," Proceedings of the National Academy of Sciences, vol. 56, no. 4, pp. 1080-1086, 1966.
[6] Z. Liu, J. S. Ume, and S. M. Kang, "Generalized nonlinear variational-like inequalities in reflexive Banach spaces," Journal of Optimization Theory and Applications, vol. 126, no. 1, pp. 157-174, 2005.

[7] Y. L. Zhao, Z. Q. Xia, Z. Q. Liu, and S. M. Kang, "Existence of solutions for generalized nonlinear mixed variational-like inequalities in Banach spaces," International Journal of Mathematics and Mathematical Sciences, vol. 2006, Article ID 36278, 15 pages, 2006.

[8] R. Ahmad and S. Shakaib Irfan, "On generalized nonlinear variational-like inequality problems," Applied Mathematics Letters, vol. 19, no. 3, pp. 294-297, 2006.

[9] A. P. Farajzadeh, A. Amini-Harandi, D. O’Regan, and R. P. Agarwal, "New kinds of generalized variational-like inequality problems in topological vector spaces," Applied Mathematics Letters, vol. 22, no. 7, pp. 1126-1129, 2009.

[10] N. V. Hung, "Sensitivity analysis for generalized quasivariational relation problems in locally G-convex spaces," Fixed Point Theory and Applications, vol. 158, 2012.

[11] N. V. Hung and P. T. Kieu, "On the existence and essential components of solution sets for systems of generalized quasivariational relation problems," Journal of Inequalities and Applications, vol. 250, 2014.

[12] N. V. Hung, L. X. Dai, E. Köbis, and J. C. Yao, “The generic stability of solutions for vector quasi-equilibrium problems on Hadamard manifolds," Journal of Nonlinear and Variational Analysis, vol. 4, no. 3, pp. 427-438, 2020.

[13] S. S. Irfan, M. F. Khan, A. P. Farajzadeh, and A. Shafie, "Generalized variational-like inclusion involving relaxed monotone operators," Advances in Pure and Applied Mathematics, vol. 8, no. 2, pp. 109-119, 2017.

[14] J. K. Kim, A. K. Khanna, and T. Ram, “On $\eta$-generalized operator variational-like inequalities," Communications in Optimization Theory, vol. 2018, 2018.

[15] M. Tavakoli, A. P. Farajzadeh, and D. Inoan, "On a generalized variational inequality problem," Filomat, vol. 32, no. 7, pp. 2433-2441, 2018.

[16] T. Chen, S. Zou, and Y. Zhang, "New existence theorems for vector equilibrium problems with set-valued mappings," Journal of Nonlinear Functional Analysis, vol. 2019, no. 1, 2019.

[17] A. P. Farajzadeh, R. Wangkeeree, and J. Kerdkaew, "On the existence of solutions of symmetric vector equilibrium problems via nonlinear scalarization," Bulletin of the Iranian Mathematical Society, vol. 45, no. 1, pp. 35-58, 2019.

[18] X. Q. Yang and C. J. Goh, "On vector variational inequalities: application to vector equilibria," Journal of Optimization Theory and Applications, vol. 95, no. 2, pp. 431-443, 1997.

[19] T. Xie and Z. Gong, "Variational-like inequalities for ndimensional fuzzy-vector-valued functions and fuzzy optimization," Open Mathematics, vol. 17, no. 1, pp. 627-645, 2019.

[20] H. Q. Ma, N. J. Huang, M. Wu, and D. O'Regan, “A new gap function for vector variational inequalities with an application," Journal of Applied Mathematics, vol. 2013, Article ID 423040, 8 pages, 2013.

[21] L. Xie, J. Li, and W. Yang, "Vector and ordered variational inequalities and applications to order- optimization problems on Banach lattices," Journal of Applied Mathematics, vol. 2013, Article ID 439394, 7 pages, 2013.

[22] J. C. Yao, "Applications of variational inequalities to nonlinear analysis," Applied Mathematics Letters, vol. 4, no. 4, pp. 89-92, 1991. 
[23] A. Gupta and R. D. Sarma, "A study of function space topologies for multifunctions," Applied General Topology, vol. 18, no. 2, pp. 331-344, 2017.

[24] A. Göpfert, H. Riahi, C. Tammer, and C. Zalinescu, Variational Methods in Partially Ordered Spaces, Springer-Verlag, New York (NY), 2003.

[25] M. Kisielewicz, Differential Inclusions and Optimal Control, Polish Scientific Publishers \& Kluwer Academic Publishers, 1991.

[26] R. Arens and J. Dugundji, "Topologies for function spaces," Pacific Journal of Mathematics, vol. 1, no. 1, pp. 5-31, 1951.

[27] I. V. Konnov and J. C. Yao, "On the generalized vector variational inequality problem," Journal of Mathematical Analysis and Applications, vol. 206, no. 1, pp. 42-58, 1997.

[28] K. Fan, "Some properties of convex sets related to fixed point theorems," Mathematische Annalen, vol. 266, no. 4, pp. 519537, 1984.

[29] A. Gupta, S. Kumar, R. D. Sarma, P. K. Garg, and R. Georege, "A topological approach to the generalized vector variational inequality problem," in presented in International Conference on Mathematical Analysis and its Applications (ICMAA -2019), pp. 14-16, Delhi, India, 2019.

[30] S. Kumar, A. Gupta, P. K. Garg, and R. D. Sarma, "Topological variants of vector variational inequality problem," in presented in International Conference on Sustainable Development: Modelling and Optimization (ICSDMO), pp. 16-17, Meerut, India, 2021. 